\title{
The role of specific enhancer-promoter interactions in the Drosophila Adh promoter switch
}

\author{
Victoria Corbin ${ }^{1}$ and Tom Maniatis \\ Harvard University, Department of Biochemistry and Molecular Biology, Cambridge, Massachusetts 02138 USA
}

\begin{abstract}
The Drosophila melanogaster alcohol dehydrogenase (Adh) gene is transcribed from two promoters active at different developmental stages. In this paper we show that the promoters are differentially stimulated by two enhancers, the $A d h$ larval enhancer and the $A d h$ adult enhancer. In early larval stages, the larval enhancer stimulates transcription from the proximal promoter; in late larval stages, the two enhancers act synergistically to stimulate transcription from the distal promoter; and in adults, the adult enhancer stimulates transcription from the distal promoter. To determine the basis for these enhancer-promoter interactions, we examined the effect of each enhancer on three different promoters. We found that the adult enhancer is stage specific and stimulates transcription from all three promoters. In contrast, the larval enhancer is potentially active in all stages and stimulates transcription from only two of the three promoters. These observations suggest that normal temporal expression of $A d h$ depends on the stage-specific activity of the adult enhancer and the differential response of the proximal and distal promoters to the larval enhancer.
\end{abstract}

[Key Words: Development; transcription regulation; gene expression; P element; tandem promoters]

Received April 26, 1989; revised version accepted October 3, 1989.

Alternative promoters are sometimes used to express the same gene product at different stages of development or in different cell types (for review, see Schibler and Sierra 1987). For example, the Drosophila melanogaster alcohol dehydrogenase (Adh) gene is transcribed from two promoters that are active during different stages of Drosophila development (Benyajati et al. 1983; Savakis et al. 1986). The proximal promoter is active in embryogenesis and early larval development, is switched off during the late third-instar larval stage, and is switched back on at a low level in adults (Fig. 1A,B). In contrast, the distal promoter is off during early larval development and is switched on briefly from late third-instar larval through early pupal stages. Both promoters are virtually inactive during the rest of pupal development. Just prior to eclosion, however, transcription from the distal promoter increases rapidly and remains at high levels throughout adult life. The same pattern of promoter switching occurs when a cloned 11.8 -kb chromosomal DNA fragment containing the Adh gene is introduced into the Drosophila germ line /Goldberg et al. 1983).

Additional germ line transformation studies have identified two regulatory elements necessary for correct $A d h$ gene expression during development (Posakony et al. 1985). The first, called the larval enhancer, is required for maximal levels of $A d h$ expression in larvae and was localized between -5000 and $-660 \mathrm{bp}$ of the distal pro-

'Current address: Rockefeller University, New York, New York 10021 USA. moter (Posakony et al. 1985; Corbin and Maniatis 1990). The second, called the adult enhancer, is required for maximal levels of $A d h$ expression in adults and was localized between -660 and -69 bp of the distal promoter (Posakony et al. 1985).

In this paper, we show that the two Adh enhancers differ from one another in two important ways. First, the adult enhancer is stage specific, whereas the larval enhancer is not. When linked to a 'neutral' $h s p 70$ promoter, the adult enhancer stimulates transcription only in third-instar larvae and adults. In contrast, the larval enhancer stimulates transcription in all developmental stages examined. Second, the adult enhancer can stimulate transcription from both $A d h$ promoters (Posakony et al. 1985) and the $h s p 70$ promoter, whereas the larval enhancer cannot directly stimulate transcription from the distal promoter. In a previous study we showed that the (downstream) proximal promoter is turned off in stages where the (upstream) distal promoter is active by transcriptional interference (Corbin and Maniatis 1989). Taken together, our results suggest that the stage-specific expression of the Adh promoters is controlled by the temporal specificity of the adult enhancer and by the differential response of the proximal and distal promoters to the larval enhancer.

Results

Experimental strategy

We identified the cis-acting DNA sequences required for stage-specific transcription from the distal and proximal 


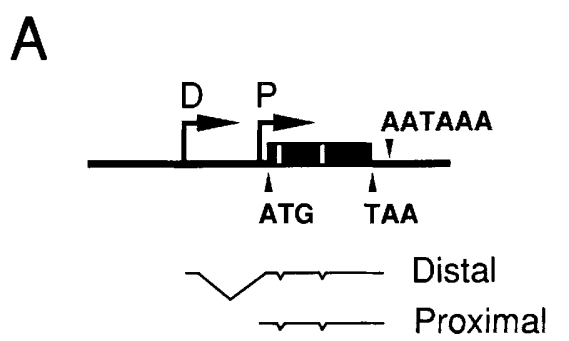

B

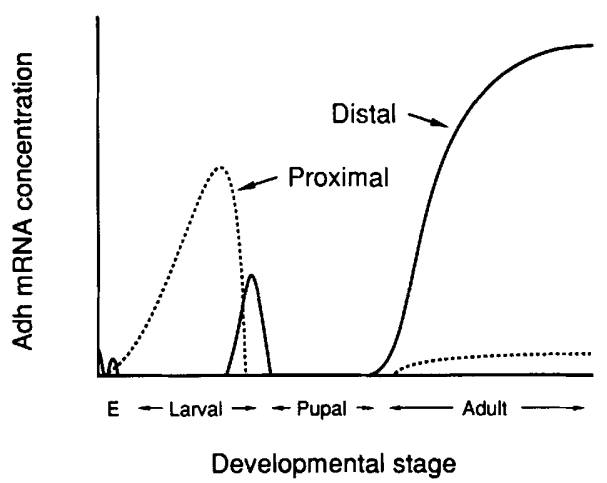

Figure 1. Temporal expression of the D. melanogaster Adh distal and proximal promoters. (A) A diagram of the D. melanogaster Adh gene. The sites of transcription initiation from the distal $(\mathrm{D})$ and proximal $(\mathrm{P})$ promoters are marked with arrows. The distal promoter is $713 \mathrm{bp}$ upstream of the proximal promoter, and both transcripts encode the same protein product. The polyadenylation signal, AATAAA, common to the two transcripts and the translation start and stop codons of the $A d h$ protein-coding region (solid boxes), ATG and TAA, are indicated. The structures of the distal and proximal transcripts are sketched below the diagram. Introns are marked by indentations. (B) The approximate levels of proximal (dotted line) and distal (solid line) $A d h$ transcripts are plotted as a function of developmental stage (from Savakis et al. 1986). Adh mRNA concentrations are given in arbitrary units.

Adh promoters by analyzing the effects of deletions or rearrangements on the expression of cloned $A d h$ genes in transgenic flies. These genes were introduced into wild-type $A d h$ embryos ( $\left.r y^{506}\right)$ or $A d h$ null embryos $\left(A d h^{f n 6}, c^{2 n} ; r^{506}\right)$. Insertions that arose from injections in the $r y^{506}$ strain were subsequently crossed into the $A d h$ null background (see Methods; Rubin and Spradling 1982; Spradling and Rubin 1982). We quantitated $A d h$ transcripts from the two promoters of the introduced genes at various developmental stages by RNase protection experiments (Zinn et al. 1983). The hybridization probes were designed to distinguish between transcripts from the introduced genes and those from the recipient $A d h^{\text {fn6 }}$ genes (Fig. 3C).

\section{Identification of the larval enhancer}

The DNA sequence between -5000 and $-660 \mathrm{bp}$ of the distal promoter contains one or more regulatory elements necessary for wild-type $A d h$ expression in larvae, but not in adults (Posakony et al. 1985). To study the role of this upstream sequence in more detail, we analyzed its effect on $A d h$ expression at different developmental stages (for diagram of constructs, see Fig. 2). The presence of the upstream sequence resulted in a small (twofold) increase in the level of maternal Adh transcripts (Fig. 3A, lanes 1 and 2; 0- to 4-hr embryos). However, in 4- to 16-hr embryos, when transcription is zygotic, the upstream sequence had little effect on transcription from either the proximal or distal promoter (Fig. 3A, lanes 3-8). From late embryogenesis through the third larval instar, the upstream sequence significantly stimulated transcription from the proximal promoter (Fig. 3A, lanes 11 and 12; Fig. 3B, lanes 1-6). In third-instar larvae and early pupae, the upstream sequence also enhanced transcription from the distal promoter (Fig. 3B, lanes 5-8). In adults, however, the upstream sequence did not stimulate transcription detectably from either promoter (Fig. 3B, lanes 9 and 10). Thus, the combined effect of the upstream sequence on both Adh promoters is to stimulate transcription from late embryogenesis through larval/early pupal development.

To test whether the $-5000-b p$ to $-660-b p$ sequence is a transcriptional enhancer, we placed it at the $3^{\prime}$ end of the $A d h$ gene or in the reverse orientation at the $5^{\prime}$ end of the gene (See Fig. 2). Transformants carrying these altered genes produced wild-type levels of $A d h$ transcripts in larvae (Fig. 4, lanes 1-7) and in adults (Fig. 4, lanes $8-12$ ). To determine whether the upstream sequence could stimulate transcription from a heterologous promoter, we placed it upstream of a truncated hsp 70 promoter which, in turn, was linked to the $A d h$-coding region (see Fig. 2). In third-instar larvae, the upstream sequence stimulated transcription from the $h s p 70$ promoter $\sim 30$-fold relative to controls (Fig. 5, lanes 5 and 6 ; for discussion of other time points, see below). Thus, the -5000 -bp to -660 -bp sequence has properties characteristic of a transcriptional enhancer: It stimulates transcription in an orientation- and distance-independent fashion and from a heterologous promoter. We refer to this enhancer as the $A d h$ larval enhancer, because it is required primarily in larvae. Our attempts to localize the larval enhancer show that it consists of at least two elements located between $-5000 \mathrm{bp}$ and - 1845 bp (Corbin and Maniatis 1990).

\section{Stage-specific activity of the enhancers}

Sequences downstream of $-660 \mathrm{bp}$ are sufficient for wild-type transcription of $A d h$ in adults /see above and Posakony et al. 1985/ and contain the $A d h$ adult enhancer (D. Falb and T. Maniatis, unpubl.). To determine whether either the larval or adult enhancer is stage specific outside the context of the $A d h$ gene, we linked each enhancer to a truncated $h s p 70$ promoter. The transcription pattern of these fusion genes should reflect the specificity of the enhancer, as the truncated promoter lacks tissue- and temporal-specific control elements (Lis et al. 1983; Garabedian et al. 1986; Hiromi and Gehring 1987; Fischer and Maniatis 1988). 

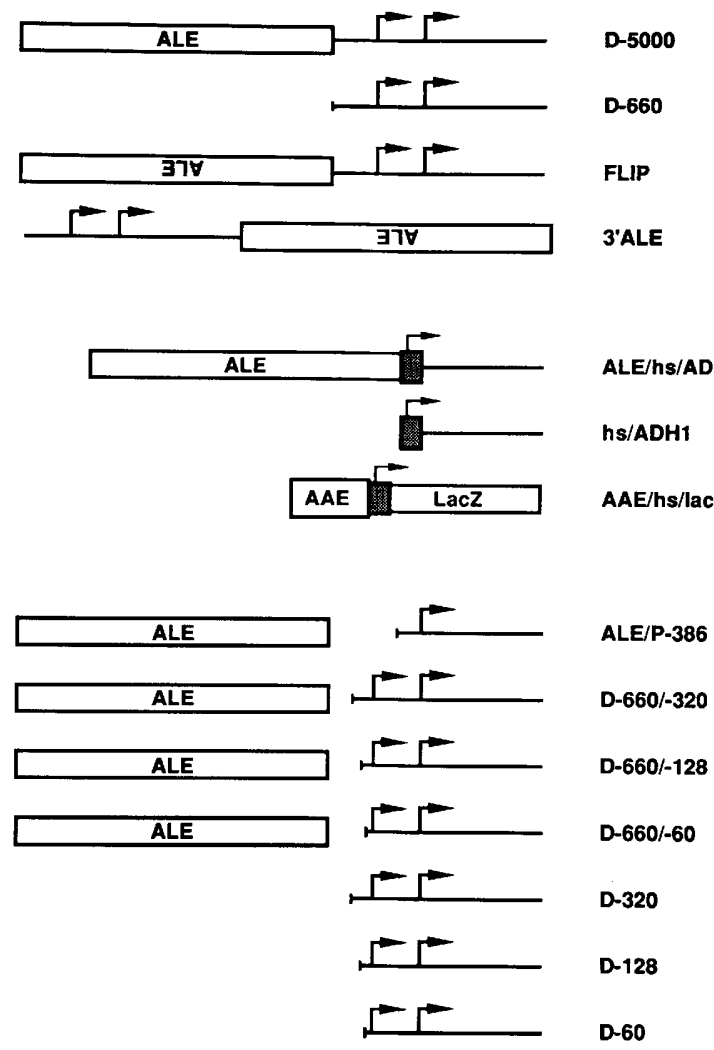

Figure 2. Structure of mutant $A d h$ genes. The structure of mutant genes (not to scale) used to identify and characterize Adh control regions is shown. The box labeled ALE /Adh larval enhancer) includes sequences between two $\mathrm{Xbal}$ sites at -660 $\mathrm{bp}$ and at approximately $-5000 \mathrm{bp}$, except in genes $A L E / h s /$ $A D H, \mathrm{pD}-660 /-320, \mathrm{pD}-660 /-128$, and $\mathrm{pD}-660 /-60$, where the ALE includes sequences between -4700 and -660 bp. The box labeled AAE (Adh adult enhancer) includes sequences from -660 to $-128 \mathrm{bp}$. The $3^{\prime}$ end points of the modified $A d h$ genes lie 640 or 1300 bp downstream of the polyadenylation signal. The presence or absence of sequences between 640 and $1300 \mathrm{bp}, 3^{\prime}$ of the polyadenylation site, does not appear to affect expression from the introduced $A d h$ genes (J. Posakony and T. Maniatis, unpubl.). The $5^{\prime}$ end points of $\mathrm{D}-5000$ and $\mathrm{pD}-660$ lie $\sim 5000$ and 660 bp upstream of the distal promoter, respectively. In FLIP, the ALE is inverted relative to the direction of $A d h$ transcription. In $3^{\prime} A L E$, the ALE is inverted and placed at the $3^{\prime}$ end of the coding region, 640 bp downstream of the polyadenylation site. The fusion gene $A L E / h s / A D H$ contains the ALE linked to the $h s p 70$ promoter $(-68$ to $+198 \mathrm{bp})$ which, in turn, is linked to the $A d h$-coding region at +9 relative to the proximal promoter initiation site. The truncated hsp70 promoter contains one copy of the heat shock consensus sequence but is not induced by heat treatments. (see Dudler and Travers 1984; Corbin 1989). The hs/ADH fusion gene was described previously (Fischer and Maniatis 1988) and contains -68 to $+198 \mathrm{bp}$ of the $h s p 70$ gene fused to the $A d h$-coding region at +9 of the proximal promoter. The $A A E / h s /$ lac $Z$ gene contains, from $5^{\prime}$ to $3^{\prime}$, Adh sequences from -660 to -128 bp of the distal promoter and a truncated $h s p 70$ promoter (from -43 to $+265 \mathrm{bp}$ of the transcription initiation site), fused in frame to the bacterial lac $Z$ gene at amino acid 9 of the protein-coding sequence. $A A E / h s / l a c Z$ transformants were a gift from D. Falb. The $A L E / P-386, D-660 /-320, D-660 /-128$, and $D-660 /-60$ genes lack sequences between $-660 \mathrm{bp}$ of the distal promoter and either $-386 \mathrm{bp}$ of the proximal promoter or $-320,-128$, or -60 bp of the distal promoter, respectively. $A L E / P-386$ and $D-660 /-60$ were described previously (Corbin and Maniatis 1989). The $D-320, D-128$, and $D-60$ genes lack sequences upstream of $-320,-128$, and $-60 \mathrm{bp}$, respectively. All of the $A d h$ inserts were cloned into the transformation vector, Carnegie 20, except pFLIP, which was cloned into the transformation vector pPl (a gift of J. Posakony). The $A d h$ deletion genes were inserted into the $\mathrm{P}$ element in the opposite orientation of the rosy (ry) gene, whereas the fusion genes were inserted into the $\mathrm{P}$ element in the same orientation as the ry gene. In most cases, several independent transformed lines were analyzed for each introduced gene: $D-5000,5$ lines; $D-660,4$ lines; $F L I P, 2$ lines; 3'ALE, 2 lines; $A L E / h s / A D H, 2$ lines; hs/ADH1, 2 lines; $A A E / h s / l a c Z, 2$ lines; $A L E / P-386,6$ lines; $D-660 /-320,3$ lines; $D-660 /-128,3$ lines; $D-660 /-60,3$ lines; $D-320,3$ lines; $D-128,3$ lines; and $D-60,3$ lines. Transcription from a given $A d h$ construct did not generally vary by more than 3 -fold between independent transformed lines; however, one $D-5000$ line produced levels of transcripts $\sim 12$-fold lower than the average (data not shown). This variability is presumably caused by the influence of nearby sequences or chromatin structure (Spradling and Rubin 1983).

Transcriptional analysis of the fusion genes showed that the adult enhancer is stage specific (Fig. 6). In firstinstar larvae, the adult enhancer did not stimulate transcription from the $h s p 70$ promoter (lane 1); in second-instar larvae, it stimulated transcription very weakly (lane 2 ; a faint band is visible after along exposure but cannot be seen in this reproduction); and in third-instar larvae it stimulated transcription to relatively high levels (lane 3). The adult enhancer did not stimulate transcription in pupae, (lanes 4 and 5), but it stimulated transcription to very high levels in adults (lanes 6 and 7). These data suggest that the transcription factors that regulate the adult enhancer are themselves activated in a stage-specific fashion. Note that the peaks in activity of the fusion gene coincide with the peaks in activity of the distal Adh promoter (Fig. 3B). This correlation suggests that the adult enhancer could stimulate transcription from the distal promoter in third-instar larvae, as well as in adults.
In contrast to the adult enhancer, the larval enhancer stimulated transcription from an $h s p 70$ promoter at all developmental stages examined (Fig. 5)-albeit to different levels at different stages (for further details, see legend to Fig. 5). This result is surprising because the larval enhancer stimulates transcription only in embryonic and larval stages in its normal context (Fig. 3A,B). These results show that the ability of an enhancer to stimulate transcription can be influenced by the linked promoter. We therefore examined the effect of each enhancer on transcription from each $A d h$ promoter at various developmental stages (see below).

The larval enhancer and proximal promoter are sufficient for wild-type transcription in early larval stages

Adh transcripts initiate exclusively at the proximal promoter in early larval stages (Fig. 1B; Savakis et al. 1986). 


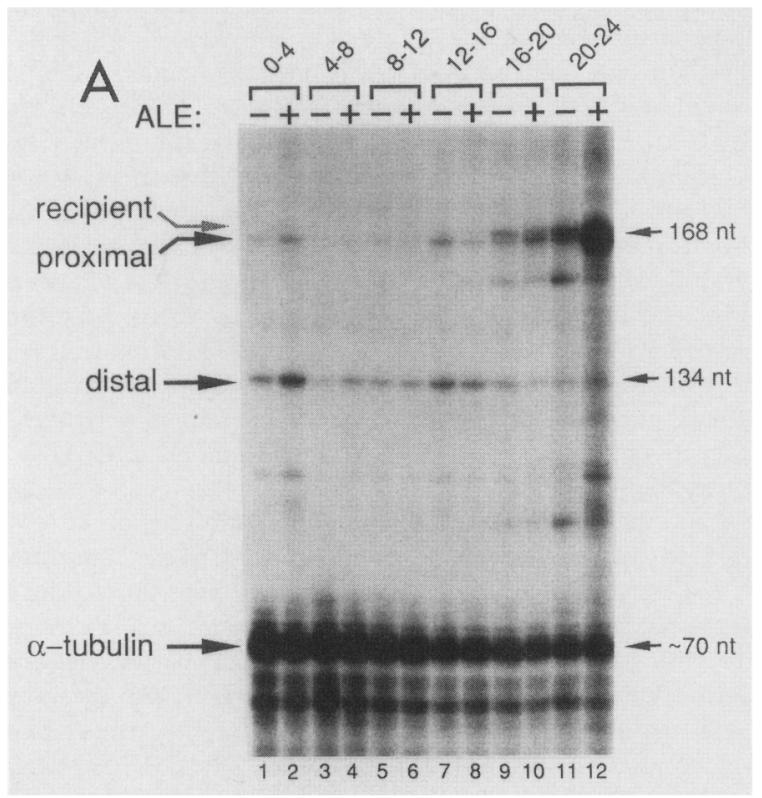

C

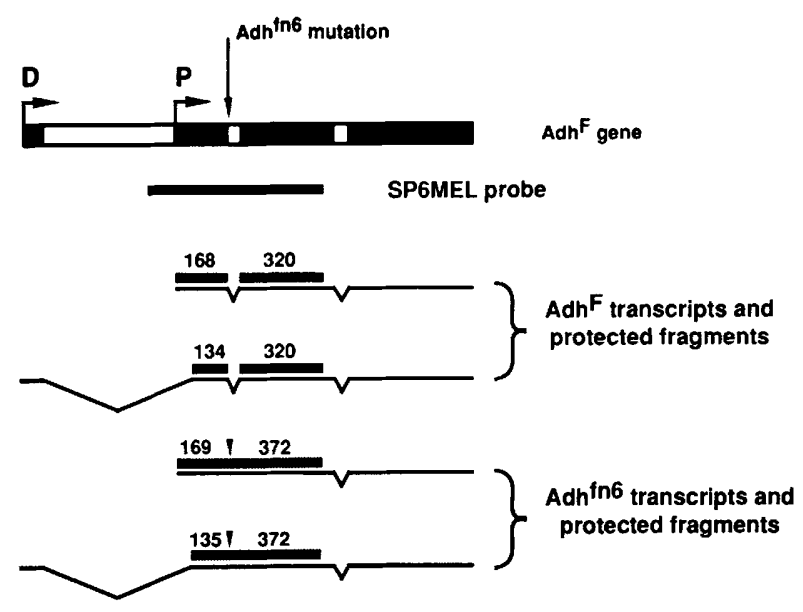

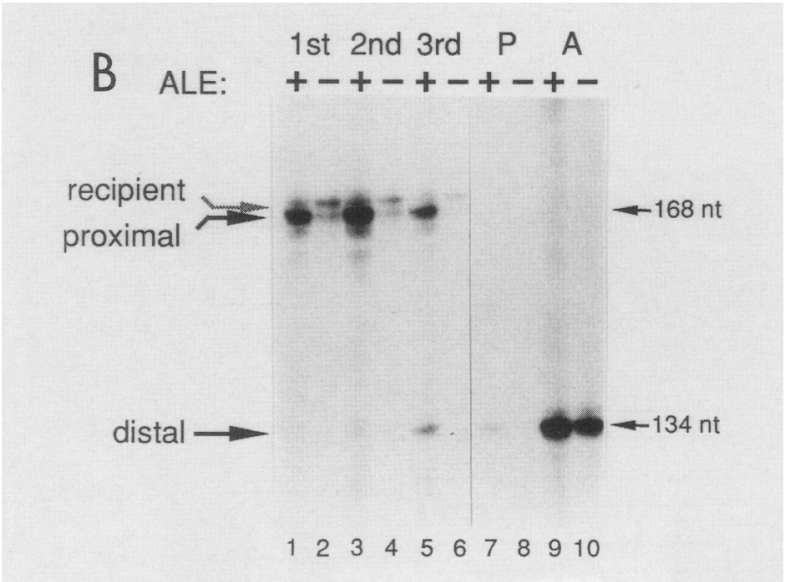

Figure 3. The $-5000-b p$ to $-660-b p$ sequence stimulates transcription from both the distal and proximal promoters in larvae. $(A)$ Preparations of total nucleic acids from embryos of representative lines transformed with $\mathrm{pD}-660$ (-lanes) or $D-5000$ (+ lanes) were analyzed by quantitative RNase mapping (see Methods). Embryos of the D-660.1 and D-5000.7 transformant lines were collected at 4 -hr intervals, as indicated above the lanes. The 0 - to 4 -hr collections measure maternal RNA, whereas the remaining collections measure zygotic RNA levels (Savakis et al. 1986). The 20- to 24-hr preparations contained some newly hatched first-instar larvae, as expected in our culture conditions. Each sample was hybridized simultaneously to the ${ }^{32}$ P-labeled, single-stranded RNA probes, SP6 $\alpha$ TUB and SP6MEL. The SP6 $\alpha$ TUB probe is complementary to $\alpha$-tubulin transcripts and protects a band of $\sim 70$ nucleotides from RNase digestion. The SP6MEL probe is complementary to $A d h$ transcripts and is described in $C$. Following RNase digestion, the products were fractionated on a $5 \%$ denaturing polyacrylamide gel and visualized by autoradiography. Transcripts from the introduced $A d h$ genes are marked by solid arrows, whereas transcripts from the $A d h^{\text {fno }}$ gene of the recipient line are marked by shaded arrows (see $C$ ). Transcripts from the distal promoter of the $A d h^{\text {fno }}$ gene are too faint to detect in the exposure shown. Similar data were obtained from independent lines transformed with the same P-element constructs. $(B)$ Preparations of total nucleic acids from larvae, pupae, and

adults of representative lines transformed with $D-5000(+$ lanes) or $\mathrm{pD}-660$ (-lanes) were analyzed by quantitative RNase mapping, as in $A$, except that only the SP6MEL probe was used (described in C). Preparations were from D-5000.7 and D-660.1 transformants during the following stages: first instar (lanes 1 and 2), second instar (lanes 3 and 4), mid-third instar (lanes 5 and 6), pupae (lanes 7 and 8 , and adult males (lanes 9 and 10). Similar data were obtained from independent lines transformed with the same P-element construct. $(C)$ Expected products from RNase mapping experiments using the $A d h$-specific probe, SP6MEL. The transcribed portion of the $A d h^{F}$ gene is shown. Solid boxes indicate exons; open boxes, introns. The extent of the complementary single-stranded RNA probe. SP6MEL, is indicated by the shaded bar. The two bracketed drawings show the RNase-resistant hybrids that form between the probe and the proximal and distal transcripts of the transformed $\left(A d h^{F}\right)$ and endogenous $\left(A d h^{f n 6}\right) A d h$ genes. Transcripts from the $A d h^{F}$ gene give rise to three fragments after RNase digestion: one of 320 nucleotides, common to transcripts from the proximal and distal promoters; one of 168 nucleotides, specific to transcripts from the proximal promoter; and one of 134 nucleotides, specific to transcripts from the distal promoter. Transcripts from the endogenous $A d h^{\text {fr6 }}$ gene are not spliced properly due to a 4-bp substitution plus 6-bp deletion near the end of the first coding exon and are unstable, present at only 5-10\% the steady-state level of wild-type Adh transcripts (Benyajati et al. 1982). Transcripts from the distal $A d h^{f n 6}$ promoter yield fragments of 135 and 372 nucleotides; transcripts from the proximal promoter, fragments of 169 and 372 nucleotides. For simplicity, most of the figures presented in this paper show only a subset of the fragments protected with this probe. 


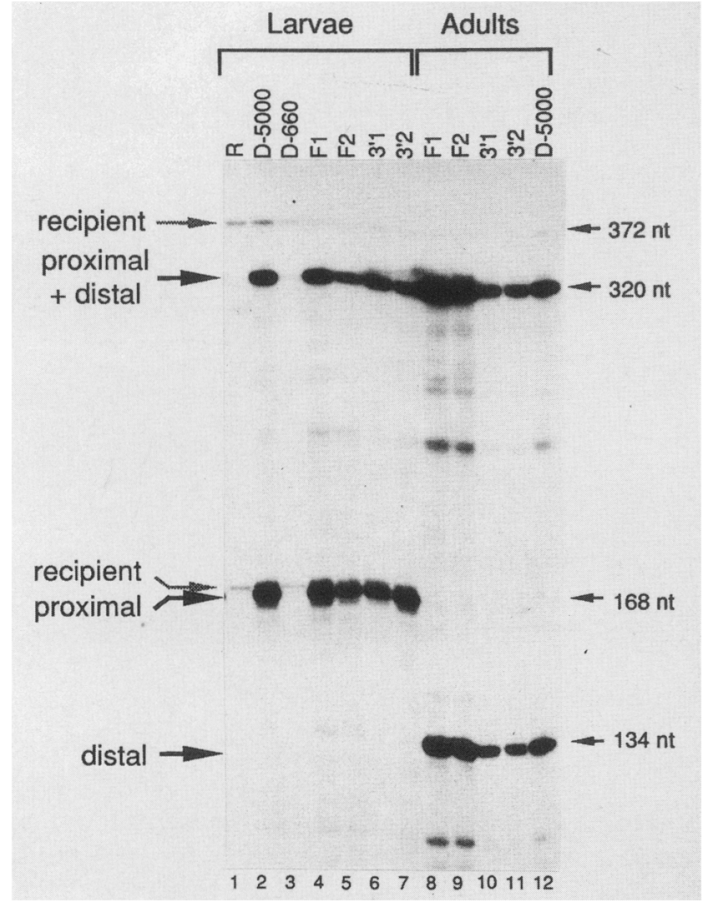

Figure 4. The -5000 -bp to -660 -bp sequence contains a transcriptional enhancer. Adh transcripts in early third-instar larvae (lanes 1-7) and adults (lanes 8-12) of the independent transformed lines D-5000.7 (lanes 2 and 12), D-660.1 (lane 3), FLIP.1 (lanes 4 and 8), FLIP.2 (lanes 5 and 9), 3'ALE.1 (lanes 6 and 10), 3'ALE.2 (lanes 7 and 11) or the recipient line $A d h^{\text {fn6 }}$, $c n$; $r y^{506}$ (lane 1) were analyzed by quantitative RNase mapping, using the probe SP6MEL (see Fig. 3C). Nucleic acids were isolated from third-instar larvae within $4 \mathrm{hr}$ of the second/third instar molt to assure that proximal transcripts were at peak levels. The distal promoter is not active this early in third larval instar. In lanes $8-12$, the faint band specific to the distal promoter of the recipient $A d h$ gene is obscured by the band specific to the distal promoter of the introduced $A d h$ gene. However, the band specific to the second exon of the recipient gene transcript can be seen at the top. (Left) Relevant bands are marked.

Our results with the $h s p 70$ promoter constructs suggest that the proximal promoter is stimulated entirely by the larval enhancer at these stages (Figs. 5 and 6). We showed that the larval enhancer and proximal promoter are sufficient for wild-type $A d h$ transcription in larvae, by fusing the larval enhancer to the proximal promoter at -386 bp (see Fig. 2). In larvae transformed with the resulting constructs, the proximal promoter was active at wild-type levels (Fig. 7, cf. lanes 1-5, with 6-7).

\section{The larval enhancer does not act directly on the distal promoter in larvae}

Our experiments with the $h s p 70$ promoter showed that both enhancers are active in third-instar larvae (Figs. 6 and 7). As shown above, the larval enhancer is required for wild-type transcription from the distal promoter at this stage, because transcription levels decrease when the larval enhancer is deleted (Fig. 3B, lanes 5 and 6). To test whether it is sufficient to stimulate the distal promoter, we placed the larval enhancer directly upstream of the TATA box (at -60 bp; see Fig. 2). This construct did not produce detectable amounts of transcription from the distal promoter in third-instar larvae (Fig. 8, lanes $6-10$ ), suggesting that additional regulatory elements are required. To localize these additional elements, we placed the larval enhancer at -128 and -320 $\mathrm{bp}$ of the distal promoter. The addition of these sequences did not increase transcription from the distal promoter (lanes 11-20). Thus, in third-instar larvae, sequences between -320 and $-660 \mathrm{bp}$ are necessary for wild-type transcription from the distal promoter. These data suggest that the adult enhancer and/or a closely associated upstream promoter element acts synergistically with the larval enhancer to stimulate transcription from the distal promoter in larvae.

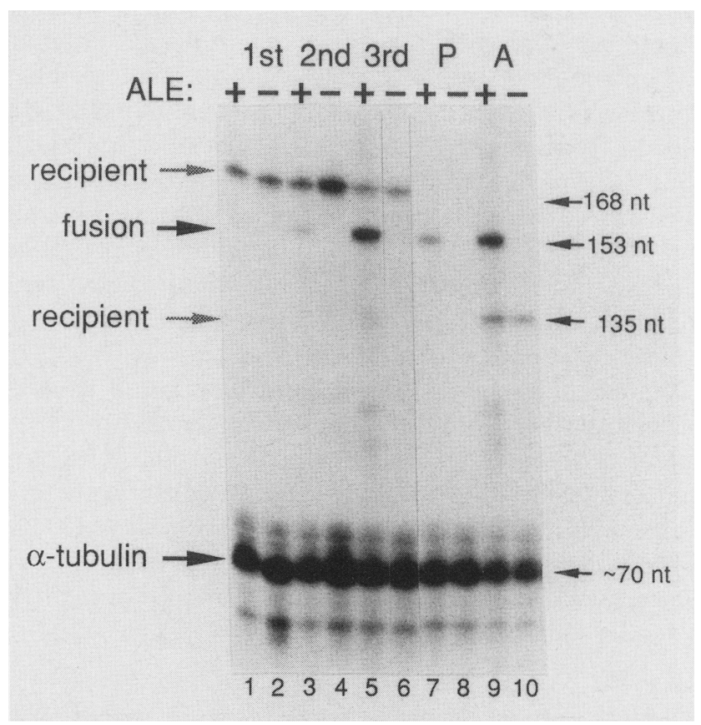

Figure 5. The larval enhancer is active throughout development. The larval enhancer stimulates transcription from the truncated $h s p 70$ promoter at all developmental stages examined, albeit to different levels in different stages. Transcription levels from the $A L E / h s / A D H$ gene (+ lanes) were compared to transcription levels from the control $h s / A D H$ gene (- lanes) by quantitative RNase mapping. Total nucleic acids were isolated from ALE/hs/ADH.1 and hs/ADH.1 transformants as first-instar larvae (lanes 1 and 2); second-instar larvae (lanes 3 and 4); third-instar larvae (lanes 5 and 6); pupae, $\sim 2$ days after white prepupa stage (lanes 7 and 8 ); and adults (lanes 9 and 10). Each sample was hybridized simultaneously to the ${ }^{32} \mathrm{P}$-labeled, single-stranded probes SP6MEL and SP6aTUB, complementary to the $A d h$ and $\alpha$-tubulin transcripts (see Methods). Following RNase digestion, the products were fractionated on a $5 \%$ denaturing polyacrylamide gel and visualized by autoradiography. (Left) Relevant bands are marked. Although data for only one line of each genotype are shown, similar results were obtained with independent transformed lines. The peaks in larval enhancer activity occur in third-instar larvae and adults, the same stages where adult enhancer activity peaks (see Fig. 6). 


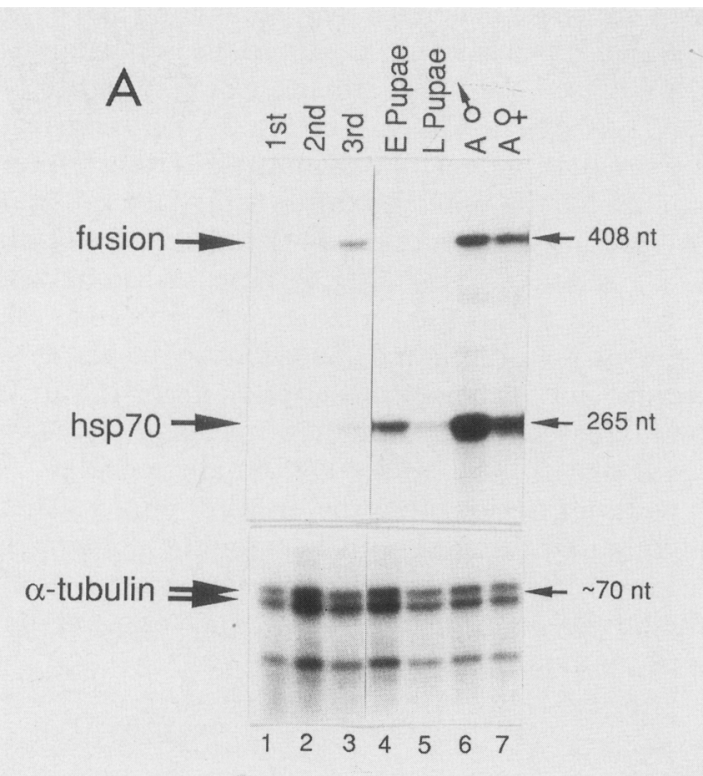

B AAE/hs/lacZ

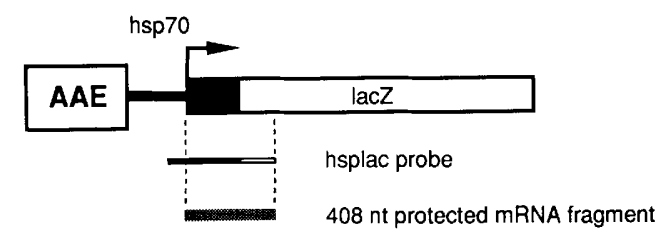

endogenous hsp70 genes

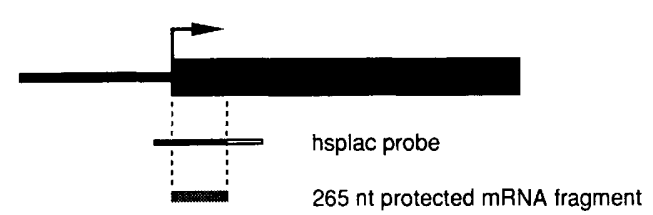

Figure 6. The adult enhancer is active in late larval stages and in adults. The effect of the adult enhancer on transcription from the truncated $h s p 70$ promoter is shown at various developmental stages. $(A)$ Quantitative RNase mapping was used to measure transcription from the $A A E / h s / l a c Z$ gene during the indicated developmental stages. Total nucleic acids were isolated from AAE/hs/lacZ.4 transformants (provided by D. Falb) during the indicated stages and hybridized simultaneously to ${ }^{32}$ P-labeled, single-stranded probes SP6-hsplac (provided by J. Fischer; Fischer et al. 1988) and SP6 $\alpha$ TUB, which are complementary to the fusion transcript and to $\alpha$-tubulin, respectively. (Left) Relevant bands are marked. Transcripts specific to the fusion gene are detected in both second- and third-instar larvae. However, the signal in second-instar larval samples is relatively weak and is difficult to see on the exposure shown. We found that the $h s / l a c Z$ fusion transcripts generally give weak signals relative to wild-type $A d h$ (data not shown). However, the $A A E / h s / l a c Z$ lines stain intensely when treated with histochemical stain specific for $\beta$-galactosidase activity (D. Falb and T. Maniatis, unpubl.). These observations suggest that the $A A E / h s / l a c Z$ gene is transcribed vigorously but that the transcripts are unstable relative to wild-type $A d h$ transcripts. The stage-specific staining pattern observed in the transformants (data not shown) parallels the stage-specific peaks in transcription shown here. $(B)$ Expected products from RNase mapping experiments using the SP6-hsplac probe. The structures of the transforming $A A E / h s / l a c Z$ gene and the endogenous hsp 70 genes are shown. The adult enhancer is shown as a large rectangle labeled AAE, hsp70 sequences are shown as solid lines (narrow and wide lines represent sequences $5^{\prime}$ and $3^{\prime}$ of the transcription initiation site, respectivelyl, and the lacZ sequence is shown as an open rectangle. The antisense SP6-hsplac probe is shown below each gene. The open and solid portions of the probe represent regions complementary to the $l a c Z$ and $h s p 70$ portions of the fusion gene transcripts, respectively. Transcripts from the endogenous $h s p 70$ genes protect 265 nucleotides of the probe, whereas transcripts from the $A A E / h s / l a c Z$ gene protect 408 nucleotides of the probe, as indicated by shaded bars (see Fischer et al. 1988).

\section{The larval enhancer does not act on the distal promoter in adults}

The larval enhancer stimulates transcription in adults when placed immediately upstream of either the $h s p 70$ or proximal Adh promoters (Fig. 5; Posakony et al. 1985). We wondered whether the larval enhancer could also stimulate transcription from the distal promoter in adults. The larval enhancer is not required in adults (Fig. 3B), but its ability to stimulate the distal promoter may be masked if the adult enhancer is sufficient for maximal transcription. To test this possibility, we deleted the adult enhancer and examined the effect of the larval enhancer on transcription from the distal promoter.

Transcription from the distal promoter decreased when the adult enhancer was deleted, as predicted (Fig. 9). Placing the larval enhancer upstream of these truncated genes did not increase the transcription levels (Fig. 9, cf. lanes $13-15$ with $16-18 ; 7-9$ with $10-12$; $1-3$ with 4-6). Thus, the larval enhancer cannot substitute for the adult enhancer in adults. Taken together with the observation that the larval enhancer is not sufficient to stimulate the distal promoter in third-instar larvae, these data suggest that the larval enhancer does not act directly on the distal promoter at any stage of development.

\section{Discussion}

We have shown that stage-specific $A d h$ expression is regulated by interactions between two promoters and two enhancers. The enhancers differ from one another in two important respects. First, the adult enhancer is active only in late larvae and adults, whereas the larval enhancer is potentially active in all stages. Second, the adult enhancer can directly stimulate transcription from both Adh promoters, whereas the larval enhancer cannot.

At present, we do not understand why the larval enhancer fails to stimulate the distal promoter. The simplest explanation is that the proteins bound at the distal 


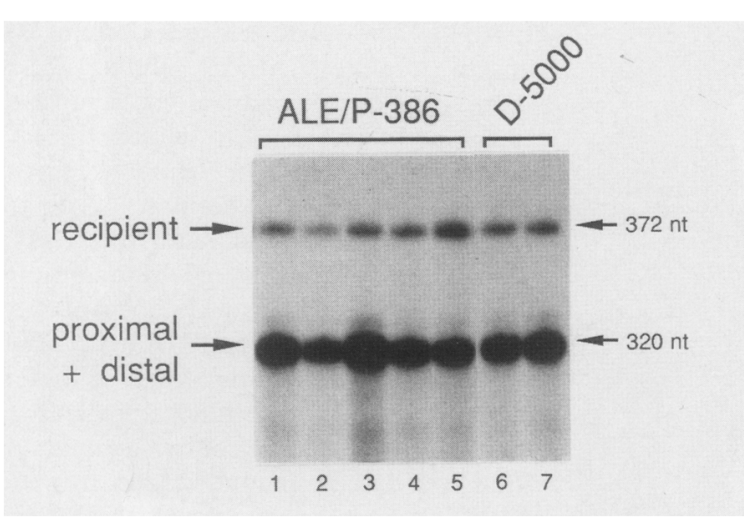

Figure 7. The larval enhancer and proximal promoter are sufficient for wild-type transcription in early larval stages. The level of $A d h$ transcripts in early third-instar larvae transformed with $A L E / P-386$ was measured by quantitative RNase mapping. Each lane shows RNA isolated from an independent transformed line. Total nucleic acids were isolated $3^{1 / 2}$ days ael and hybridized to the ${ }^{32} \mathrm{P}$-labeled, single-stranded probe, SP6MEL, complementary to Adh transcripts. Following RNase digestion, the products were fractionated on a $5 \%$ denaturing polyacrylamide gel and visualized by autoradiography. (Left) Relevant bands are marked. (Lanes 1-5) Independent lines transformed with $A L E / P-386$; (lanes 6 and 7 ) independent lines transformed with the full-length control gene, $D-5000$.

promoter cannot interact functionally with the proteins bound at the larval enhancer. Several lines of evidence suggest that the restrictive proteins act, either directly or indirectly, through the TATA box of the distal pro- moter. First, the $h s p 70$ and proximal Adh promoters, which both respond to the larval enhancer, do not appear to share any common regulatory sequences other than the TATA box motif, TATAAATA. In contrast, the nonresponsive distal promoter contains a different TATA box motif, TATTTAA. Second, the distal promoter remains unresponsive to the larval enhancer even when upstream promoter sequences are added, whereas the proximal Adh promoter remains responsive even when promoter sequences upstream of -80 are deleted. Furthermore, the larval enhancer also fails to stimulate transcription from a truncated sgs-3 promoter (TATA box sequence TATAAAAAG), whereas control enhancers do stimulate transcription (V. Corbin and R. Cohen, unpubl.). These data suggest that the TATA box, rather than an upstream promoter element, is important for interactions between the larval enhancer and the linked promoter. We therefore propose that different TATA factors bind to the proximal and distal Adh promoters or that the same factors bind differently to the two TATA motifs. In either case, only the proximal promoter factor(s) can interact with the factors bound at the larval enhancer.

This interpretation is supported by two different kinds of observations. First, in vitro DNA binding studies suggest that enhancer and TATA-binding proteins interact. In the presence of enhancer-binding proteins, the transcription factor IID (TFIID) binds more extensively to the TATA box (Sawadogo and Roeder 1985; Horikoshi et al. 1988a,b|. Second, in vivo studies suggest that certain classes of TATA- and enhancer-binding proteins cannot

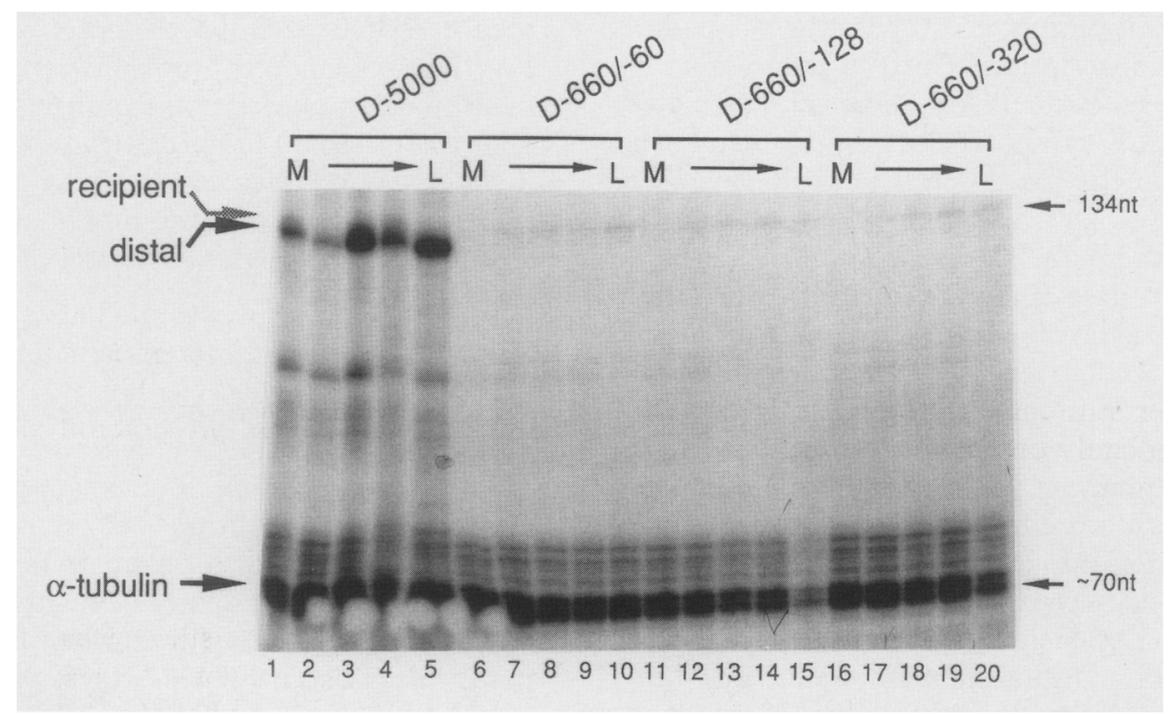

Figure 8. The larval enhancer does not act directly on the distal promoter in third-instar larvae. Transcription from the distal promoter of $A d h$ genes that lack the adult enhancer was measured in mid- to late-third instar larval transformants by quantitative RNase mapping using the SP6MEL and SP6 $\alpha$ TUB probes (see Fig. 3C). Samples from the indicated transformed lines were collected at 5 -hr intervals from the middle to the late part of the third larval instar to ensure that the peak in distal promoter activity was not missed because of small differences in the rate of development between different transformed lines. Total nucleic acids were isolated from third-instar larvae $15 \pm 2 \mathrm{hr}$ (lanes 1,6,11, and 16), $20 \pm 2 \mathrm{hr}$ (lanes 2, 7, 12, and 17), $25 \pm 2 \mathrm{hr}($ lanes 3, 8, 13, and 18), 30 $\pm 2 \mathrm{hr}$ (lanes 4, 9, 14, and 19) and $35 \pm 2 \mathrm{hr}$ (lanes 5, 10,15, and 20) after the second- to third-instar molt. In the exposure shown, the strong bands specific to transcripts initiated at the distal promoter of the $D-5000$ transformants (lanes 1-5) obscure the weak bands specific to the recipient $A d h$ gene. The recipient $A d h$ gene was expressed at equivalent levels in all lanes (data not shown). Similar data were obtained from independent lines transformed with the same P-element vectors. 


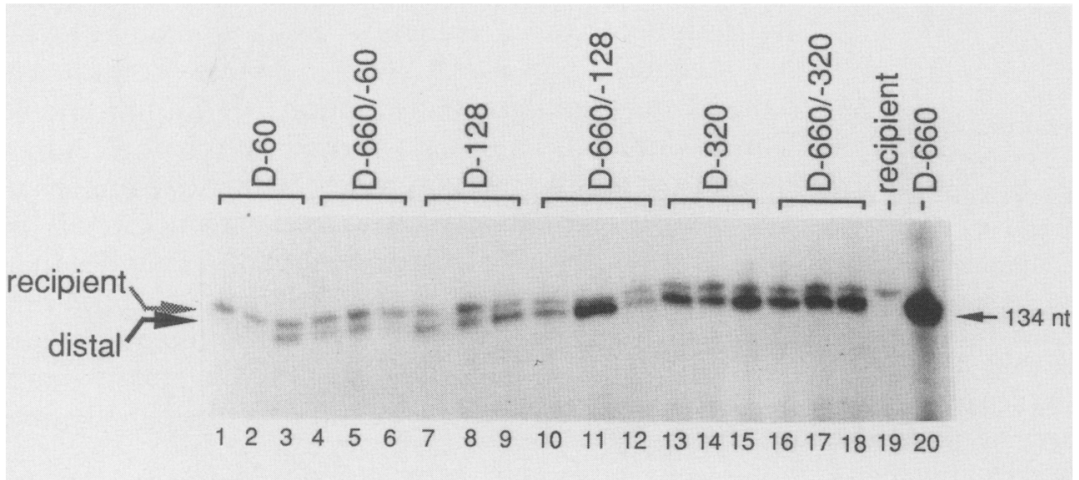

Figure 9. The larval enhancer cannot compensate for the AAE in adults. To test whether the larval enhancer could substitute for the adult enhancer in adults, the larval enhancer was placed at different locations upstream of the distal promoter. Adh transcripts from transformed adult flies carrying these genes were measured by quantitative RNase mapping, using the SP6MEL probe (Fig. 3C). Each lane shows RNA isolated from an independent transformed line of the indicated genotype (Left) Relevant bands are marked. For simplicity, only fragments specific to the distal promoters of the introduced and recipient genes are shown. The proximal promoters of all of the introduced genes are essentially off in adults, except in two of the $D-660 /-60$-transformed lines, in which the proximal promoters are active at levels approximately threefold higher than those of wild type. The proximal promoter may be slightly derepressed in these lines because readthrough transcription from the weakened distal promoter does not efficiently interfere with initiation at the proximal promoter (Corbin and Maniatis 1989).

interact. For example, certain combinations of TATA motifs and enhancer elements fail to stimulate transcription in yeast (Chen and Struhl 1989). Moreover, the adenovirus E1A gene product selectively stimulates mammalian and viral promoters containing a specific TATA box sequence (Simon et al. 1988). Of the enhancer-promoter combinations tested here, only the larval enhancer/distal promoter combination seems unable to stimulate transcription. Thus, in the context of the wild-type $A d h$ gene, the larval enhancer stimulates only the proximal $A d h$ promoter, leaving the distal promoter dependent on the (stage-specific) adult enhancer for stimulation.

An alternative explanation for our results is that both enhancers can stimulate transcription from the distal promoter but that the larval enhancer failed to do so because the distal promoter lacked an essential upstream promoter element in our constructs. This hypothetical element would facilitate interactions between the distal promoter and any enhancer and, together with the distal promoter TATA box, would provide a functional target for enhancer interactions. Because the adult enhancer can stimulate the distal promoter when sequences between -387 and -128 bp are deleted (U. Heberlein and R. Tjian, pers. comm.), this element would map upstream of -387 and downstream of the adult enhancer (currently localized between -660 and -450 bp; D. Falb and T. Maniatis, unpubl.). By this view, the distal and proximal promoter TATA boxes again appear to be functionally distinct, because the proximal, but not the distal, TATA box is apparently sufficient to respond to the larval enhancer.

Another explanation for our results is that the larval enhancer preferentially interacts with the proximal promoter and therefore fails to interact with the distal pro- moter in our constructs. Such competition for an enhancer occurs in the chicken $\epsilon$-globin locus, where an enhancer located between the adult $\beta$-globin and the embryonic $\epsilon$-globin genes preferentially interacts with the $\beta$-globin promoter in adults (Choi and Engel 1988). Although an analogous mechanism cannot be ruled out for the Adh locus, we note that in the constructs examined here, the proximal promoter is active only at very low levels in adults, probably because low levels of readthrough transcription from the distal promoter prevent the proximal promoter from becoming fully active (Corbin and Maniatis 1989). Under these circumstances, it seems unlikely that the proximal promoter could effectively compete with the distal promoter.

\section{A model for Adh promoter switching}

A model for the stage-specific regulation of the two $A d h$ promoters is presented in Figure 10. During larval development, transcription from the proximal promoter is stimulated by interactions between factors bound to the larval enhancer and to elements near the transcription start site. These factors presumably interact through a DNA looping mechanism (Ptashne 1986). The distal promoter is quiescent at this stage, probably because the transcription factors that bind to the adult enhancer are absent or inactive.

In late third-instar larvae, transcription from the distal promoter is stimulated by the synergistic action of both enhancers. We propose that the larval enhancer stimulates transcription from the distal promoter indirectly at this stage, by acting through the adult enhancer. For example, factors bound at the larval enhancer could facilitate the binding of factors to the adult enhancer. The latter factors would then interact directly with the 


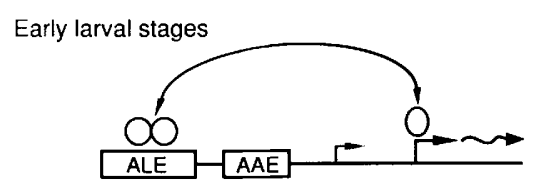

Late larval stages

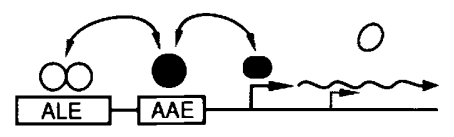

Adults

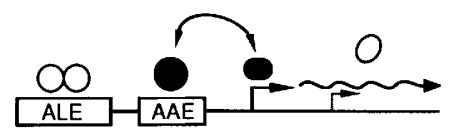

Figure 10. Model of $A d h$ enhancer-promoter interactions through development. A diagram illustrating a model for interactions between transcription factors bound to the larval enhancer (ALE), the adult enhancer (AAE), and distal and proximal promoters at three developmental stages. The larval enhancer factors (open circles) and proximal promoter factors are present throughout larval development and in adults lopen ovals). In contrast, the adult enhancer and distal promoter factors are present only in mid to late third-instar larvae and in adults (solid circles). In first through early third-instar larval stages, factors bound to the larval enhancer and proximal promoter interact to stimulate transcription. In late third instar, the distal promoter is stimulated by transcriptional activators bound to both the larval enhancer and to the adult enhancer or a closely associated element. The transcription factors that had been bound to the proximal promoter are destabilized by readthrough transcription from the distal promoter and presumably fall off the DNA (Corbin and Maniatis 1989). In adults, the transcription factors for all four regulatory elements are present, but only those bound to the AAE and distal promoter interact. The activity of the proximal promoter is significantly decreased, but not turned off completely, by transcriptional interference (Corbin and Maniatis 1989), and the larval enhancer does not interact with the distal promoter, apparently because the factors are incompatible.

factors bound at the distal promoter to stimulate transcription. As transcription levels from the distal promoter rise, the activity of the (downstream) proximal promoter is severely curtailed by transcription interference from the upstream promoter /Corbin and Maniatis 1989|.

Early in pupal development, both $A d h$ promoters are essentially switched off (Savakis et al. 1986). The mechanism of promoter inactivation is unknown. Possibly, the factors necessary for $A d h$ transcription are inactivated or absent during pupal development, or a repressor may bind and inactivate each promoter. Just prior to eclosion, the distal promoter is reactivated. Because transcription factors may be relatively scarce at this stage, both enhancers may act synergistically to stimulate transcription from the distal promoter, as they do in larvae. However, we have not yet examined this possibility in detail.

After eclosion, the activity of the distal promoter continues to increase and remains high throughout adult life. Curiously, the larval enhancer is not required for normal levels of transcription from either promoter at this stage. The adult enhancer apparently stimulates the distal promoter to maximal levels without the help of the larval enhancer. Possibly the larval enhancer is not required at this stage because the adult enhancer factors are more active or abundant in adults than in late thirdinstar larvae. As in late third-instar larvae, the activity of the proximal promoter is reduced to low levels by transcription interference (Corbin and Maniatis 1989).

In conclusion, we propose that the developmental switch from the proximal to distal promoter is controlled by three parameters: (1) the differential abilities of the two promoters to respond to the larval enhancer; (2) the stage-specific activity of the adult enhancer; and (3) transcriptional interference. Thus, both the inherent properties of $A d h$ control elements and their arrangement are important components of the Adh promoter switch.

\section{Methods}

\section{Establishment of transformed Drosophila lines}

Transformation vectors and the helper plasmid pp25.7wc (Karess and Rubin 1984) were injected into $\mathrm{ry}^{506}$ or $A d h^{f n 6}, \mathrm{cn}$; ry ${ }^{506}$ embryos, using standard procedures (Rubin and Spradling 1982); Spradling and Rubin 1982; Goldberg et al. 1983). Insertions arising from injections into $r y^{506}$ embryos were crossed into the $A d h^{f n 6}, c n ; r y^{506}$ background, as described (Fischer and Maniatis 1988). Chromosomal linkages were assigned and homozygous lines were selected as described (Goldberg et al. 1983; Fischer and Maniatis 1988). Most lines discussed in the paper are homozygous. Insertions that were homozygous lethal were kept over the balancer chromosome, TM2 (Lindsley and Grell 1968).

\section{Transformation vectors}

All Adh fragments originated from plasmid sAF2, which contains the $A d h^{F}$ allele and flanking DNA (Goldberg 1980). Plasmids were constructed using standard methods (Maniatis et al. 1982) and are described briefly below. All of the modified Adh genes shown in Figure 2, except pFLIP, were cloned into Carnegie 20 (Rubin and Spradling 1983). The basic structure of the modified $A d h$ genes is shown in Figure 2. $\mathrm{pD}-660$ was made by inserting an $X$ hoI linker into the $X b a I$ site at +2510 (relative to the distal start site) in clone sAF2 and ligating the 3.2-kb XbaI-XhoI fragment into the $\mathrm{XbaI}$ and Sall sites of pC20X, a derivative of Carnegie 20 (Rubin and Spradling 1983). $\mathrm{pD}-660$ thus contains $A d h$ sequences from -660 to +2510 $\mathrm{bp}$, relative to the distal cap site and has a unique $X b a \mathrm{I}$ site at -660 bp. pD -5000 was made by inserting the $4.4-\mathrm{kb} X b a \mathrm{I}$ fragment of sAF2 (containing sequences between approximately -5000 and $-660 \mathrm{bp}$ ) into the $\mathrm{XbaI}$ site of $\mathrm{pD}-660$ and screening for insertions in the wild-type orientation. The plasmid pP-386 was made by inserting an $\mathrm{XbaI}$ linker into the HpaI site of $\mathrm{pD}-660$, cutting with $\mathrm{XbaI}$, and ligating under dilute conditions to delete $A d h$ sequences 5 ' to -386 of the proximal promoter. The 4.4-kb XbaI fragment was inserted into the 
$\mathrm{XbaI}$ site of $\mathrm{pP}-386$ to make $\mathrm{pALE} / \mathrm{P}-386$, which harbors an internal deletion for $A d h$ sequences between $-660 \mathrm{bp}$ of the distal promoter and -386 bp of the proximal promoter.

The transformation vectors $\mathrm{pD}-320, \mathrm{pD}-128$, and $\mathrm{pD}-60$ were made by ligating the DraI-XhoI, FspI-XhoI, and SalI$X$ hoI fragments, which contain $A d h$ sequences from -320 , -128 , and -60 to about +3100 , respectively, into the P-element vector, Carnegie 20 or a simple derivative. To make the plasmids $\mathrm{pD}-660 /-320, \mathrm{pD}-660 /-128$, and $\mathrm{pD}-660 /-60$, the DraI-XhoI, FspI-XhoI, and SalI-Xhol fragments described above were subcloned into pSP73 (provided by D. Melton). The inserts were then isolated as $B g / I I-X h o I$ fragments and ligated with Carnegie 20 (cut with $\mathrm{HpaI}$ and SalI) and a 4.1-kb HincIIBamHI fragment containing the larval enhancer (sequences from about -4800 to -660 ) from $p 4.4$. Plasmid $p 4.4$ was made by inserting the 4.4-kb XbaI fragment of sAF2, which contains the larval enhancer, into the $X b a I$ site of pSP64.

Plasmid R68BX was made by inserting a BgIII linker into the Asp 718 site of R68HX (a gift of J. Fischer), which contains the hsp 70 gene from -68 to $+198 \mathrm{bp}$, fused to the $A d h$-coding region at +9 of the proximal transcript. Adh sequences extended $\sim 1300$ bp past the polyadenylation site. Plasmid pALE/hs/ADH was made by ligating the BglII-XhoI fragment of R68BX to the $4.1-\mathrm{kb}$ HincII-BamHI fragment of $\mathrm{p} 4.4$ and the $10.7-\mathrm{kb} H \mathrm{HaI}-$ Sall-digested Carnegie 20 vector. The $A A E / h s p / l a c Z$ transformants were a gift of $\mathrm{D}$. Falb. The hs/ADH transformants were a gift of J. Fischer (Fischer and Maniatis 1988). Both are described in the legend to Figure 2.

pFLIP was made by modifying PTARP, which contains the 11.8-kb SacI fragment of $A d h^{F}$ within the P-element vector pPL-1 (both gifts of J. Posakony). First, pTARP was partially digested with $X b a I$ and ligated to the -5000 -bp to -660 -bp Adh fragment, such that the -5000 - to -660 -bp fragment was inserted at +2510 in the $3^{\prime}$ to $5^{\prime}$ direction relative to the remainder of the $A d h$ gene. Next, the $r y^{+}$gene, contained on an 8.1-kb SalI-cut genomic DNA fragment, was ligated into the unique $X$ hoI site to give pFLIP.

\section{RNA analysis}

Total nucleic acid was purified from staged Drosophila embryos, larvae, pupae, and adults, as described (Fischer and Maniatis 1986). Embryos were collected every $4 \mathrm{hr}$ and allowed to age at $25^{\circ} \mathrm{C}$ for varying times. Most of the embryos were of the correct developmental stage, as judged by bright-field microscopy. Larvae were loosely staged by collecting embryos for $\sim 12$ $\mathrm{hr}$ and letting them age for the appropriate amount of time: First-instar larvae were collected $36 \pm 6 \mathrm{hr}$ after egg laying (ael); second instar, $53 \pm 6 \mathrm{hr}$ ael; third instar, $78 \pm 6 \mathrm{hr}$ ael. For experiments where carefully staged third-instar larvae were needed, larvae were isolated $<4 \mathrm{hr}$ after the second- to third-instar molt and allowed to age for the appropriate amount of time, as indicated in the figure legends. Adult samples were collected 4 days after eclosion.

Quantitative RNase mapping experiments were carried out as described (Zinn et al. 1983), except that hybridizations were performed at $37^{\circ} \mathrm{C}$ without prior incubation at $85^{\circ} \mathrm{C}$, and RNase digestions were carried out at $25^{\circ} \mathrm{C}$ for $30 \mathrm{~min}$.

\section{Hybridization probes}

Continuously labeled, single-stranded RNA probes were prepared as described (Melton et al. 1984), except that BSA was added to a concentration of $60 \mathrm{mg} / \mathrm{ml}$. The plasmids pSP6MEL and SP6-hsplac were provided by J. Fischer (Fischer and Maniatis 1986; Fischer et al. 1988). The plasmid pSP6 $\alpha$ TUB was made by inserting an $\sim 400$-bp XbaI-Sall fragment of genomic DNA from pDMTal (a gift of P. Wensink) into the XbaI and Sall sites of pSP65.

\section{Acknowledgments}

We thank R. Brent, B. Cohen, T. Abel, S. Abmayr, M. Baron, and D. Falb for helpful discussions and critical reading of the manuscript. We thank U. Heberlein and R. Tjian for communicating unpublished results. We thank J. Fischer, J. Posakony, and $\mathrm{P}$. Wensink for providing plasmids, and J. Fischer providing the $h s / A D H$ transformants. We are especially grateful to $\mathrm{D}$. Falb for providing the $A A E / h s / l a c Z$ transformants and for helpful discussions of his unpublished work. This work was supported by a grant to T.M. from the National Institutes of Health.

\section{References}

Benyajati, C., A.R. Place, N. Wang, E. Pentz, and W. Sofer. 1982. Deletions at intervening splice sites in the alcohol dehydrogenase gene of Drosophila. Nucleic Acids Res. 10: 72617272.

Benyajati, C., N. Spoerel, H. Haymerle, and M. Ashburner. 1983. The messenger RNA for alcohol dehydrogenase in Drosophila melanogaster differs in its $5^{\prime}$ end in different developmental stages. Cell 33: 125-133.

Chen, W. and K. Struhl. 1989. Yeast upstream activator protein GCN4 can stimulate transcription when its binding site replaces the TATA element. EMBO /. 8: 261-268.

Choi, O.B. and J.D. Engel. 1988. Developmental regulation of $\beta$-globin gene switching. Cell 55: 17-26.

Corbin, V.L. 1989. Regulation of the proximal and distal promoters of the D. melanogaster $A d h$ gene. Ph.D. Thesis. Harvard University, Cambridge.

Corbin, V. and T. Maniatis. 1989. Role of transcriptional interference in the Drosophila melanogaster Adh promoter switch. Nature 337: 279-282.

1990. Identification of cis-regulatory elements required for larval expression of the Drosophila alcohol dehydrogenase gene. Genetics (in press).

Dudler, R. and A.A. Travers. 1984. Upstream elements necessary for optimal function of the $h s p 70$ promoter in transformed flies. Cell 38: 391-398.

Fischer, J.A. and T. Maniatis. 1986. Regulatory elements involved in Drosophila Adh gene expression are conserved in divergent species and separate elements mediate expression in different tissues. EMBO J. 5: 1275-1289.

- 1988. Drosophila Adh: A promoter element expands the tissue specificity of an enhancer. Cell 53: 451-461.

Fischer, J.A., E. Giniger, T. Maniatis, and M. Ptashne. 1988. GAL4 activates transcription in Drosophila. Nature 332: $853-856$.

Garabedian, M.J., B.M. Shephard, and P.C. Wensink. 1986. A tissue-specific transcription enhancer from the Drosophila yolk protein 1 gene. Cell 45: 859-867.

Goldberg, D.A. 1980. Isolation and partial characterization of the Drosophila alcohol dehydrogenase gene. Proc. Natl. Acad. Sci. 77: 5794-5798.

Goldberg, D.A., J.W. Posakony, and T. Maniatis. 1983. Correct developmental expression of a cloned alcohol dehydrogenase gene transduced into the Drosophila germline. Cell 34: $58-73$.

Hiromi, Y. and W.J. Gehring. 1987. Regulation and function of the Drosophila segmentation gene fushi tarazu. Cell 50: $963-974$. 
Horikoshi, M., M.F. Carey, H. Kakidani, and R.G. Roeder. 1988a. Mechanism of action of yeast activators: Direct effect of GAL4 derivatives on mammalian TFIID-promoter interactions. Cell 54: 665-669.

Horikoshi, M., T. Hai, Y.-S. Lin, M.R. Green, and R.G. Roeder. 1988b. Transcription factor ATF interacts with the TATA factor to facilitate establishment of a preinitiation complex. Cell 54: 1033-1042.

Karess, R.E., and G.M. Rubin. 1984. Analysis of P transposable element functions in Drosophila. Cell 38: 135-146.

Lindsley, D.L. and E.H. Grell. 1968. Genetic variations in Drosophila melanogaster. Carnegie Inst. Wash. Publ. 627.

Lis, J., J.A. Simon, and C.A. Sutton. 1983. New heat shock puffs and $\beta$-galactosidase activity resulting from transformation of Drosophila with an hsp70-1acZ hybrid gene. Cell 35: $403-410$.

Maniatis, T., E.F. Fritsch, and J. Sambrook. 1982. Molecular cloning: A laboratory manual. Cold Spring Harbor Laboratory, Cold Spring Harbor, New York.

Melton, D.A., P.A. Krieg, M.R. Rebagliati, T. Maniatis, K. Zinn, and M.R. Green. 1984. Efficient in vivo synthesis of biologically active RNA and RNA hybridization probes from plasmids containing a bacteriophage SP6 promoter. Nucleic Acids Res. 12: 7035-7056.

Posakony, J.W., J.A. Fischer, and T. Maniatis. 1985. Identification of DNA sequences required for the regulation of Drosophila Alcohol dehydrogenase expression. Cold Spring Harbor Symp. Quant. Biol. 50: 515-520.

Ptashne, M. 1986. Gene regulation by proteins acting nearby and at a distance. Nature 322: 697-701.

Rubin, G.M. and A.C. Spradling. 1982. Genetic transformation of Drosophila with transposable element vectors. Science 218: $348-353$.

. 1983. Vectors for P element-mediated gene transfer in Drosophila. Nucleic Acids Res. 11: 6341-6351.

Savakis, C., M. Ashburner, and J.H. Willis. 1986. The expression of the gene coding for alcohol dehydrogenase during the development of Drosophila melanogaster. Dev. Biol. 114: $194-207$.

Sawadogo, M. and R.G. Roeder. 1985. Interaction of a gene-specific transcription factor with the adenovirus major late promoter upstream of the TATA box region. Cell 43: 165-175.

Schibler, U. and F. Sierra. 1987. Alternative promoters in developmental gene expression. Annu. Rev. Genet. 21: 237-257.

Simon, C.M., T.M. Fisch, B.J. Benecke, J.R. Nevins, and N. Heintz. 1988. Definition of multiple, functionally distinct TATA elements, one of which is a target in the hsp 70 promoter for E1A regulation. Cell 52: 723-729.

Spradling, A.C. and G.M. Rubin. 1982. Transposition of cloned $\mathrm{P}$ elements into Drosophila germ line chromosomes. Science 218: $341-347$.

- 1983. The effect of chromosomal position on the expression of the Drosophila xanthine dehydrogenase gene. Cell 34: 47-57.

Zinn, K., D. DiMaio, and T. Maniatis. 1983. Identification of two distinct regulatory regions adjacent to the human $\beta$-interferon gene. Cell 34: 865-879. 


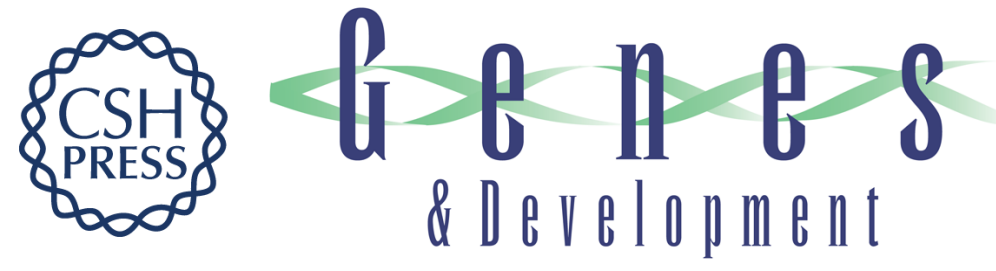

\section{The role of specific enhancer-promoter interactions in the Drosophila Adh promoter switch.}

$\checkmark$ Corbin and T Maniatis

Genes Dev. 1989, 3:

Access the most recent version at doi:10.1101/gad.3.12b.2191

References This article cites 29 articles, 4 of which can be accessed free at:

http://genesdev.cshlp.org/content/3/12b/2191.full.html\#ref-list-1

License

Email Alerting

Service

Receive free email alerts when new articles cite this article - sign up in the box at the top right corner of the article or click here.

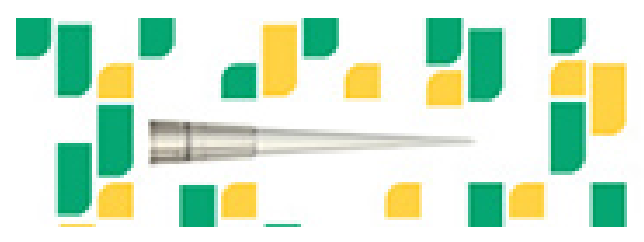

Focused on your science.

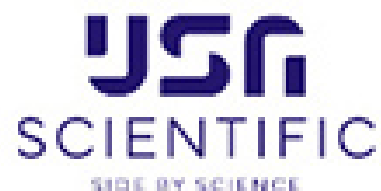

Copyright @ Cold Spring Harbor Laboratory Press 